\section{RENDIMIENTO, CALIDAD DE FRUTO Y EFICIENCIA EN EL USO DEL AGUA DEL CHILE 'MIRASOL' BAJO RIEGO DEFICITARIO}

\section{YIELD, FRUIT QUALITY AND WATER USE EFFICIENCY OF CHILI 'MIRASOL' UNDER IRRIGATION DEFICIT}

\author{
Alfonso Serna Pérez ${ }^{*}$ Jorge A. Zegbe
}

Campo Experimental Zacatecas, Instituto Nacional de Investigaciones Forestales, Agrícolas y Pecuarias. Apartado Postal No.18. 98500, Calera de V.R., Zacatecas, México. Tel.: (01) 4789850198 Ext. 308; Fax: (01) 478 9850363.

^Autor para correspondencia (aserna@zacatecas.inifap.gob.mx.)

\section{RESUMEN}

La disponibilidad de agua es la principal limitante ambiental para la producción de chile (Capsicum annuum L.) seco en el Norte Centro de México. El objetivo de este estudio fue comparar cuatro formas de riego deficitario (RD) con el riego completo (RC), con base en rendimiento, calidad del chile seco y eficiencia del uso del agua de riego (EUAR) en chile 'Mirasol' cultivado en una zona semiárida. Los tratamientos de RD fueron: 85 \% (RD 85), 70 \% (RD 70), 55 \% (RD 55), y 40 \% (RD 40) del RC. El ahorro promedio de agua en RD 85, RD 70, RD 55 y RD 40 fue de $8,16,23$ y $30 \%$, respectivamente. El tratamiento RD 40 produjo el más alto porcentaje de fruta comercial, con rendimientos similares al RC en dos de los tres años evaluados. El RD 40 incrementó la EUAR, y redujo el volumen de agua aplicada en $1520 \mathrm{~m}^{3} \mathrm{ha}^{-1}$ comparado con RC. Por tanto, este tratamiento podría ser una alternativa para el ahorro de agua en esta región semiárida y otras que enfrentan sobre-explotación de acuíferos, sin detrimento en la calidad del chile seco. No obstante, más estudios con RD para este cultivo son necesarios en áreas de baja o nula precipitación pluvial.

Palabras clave: Capsicum annuum, déficit hídrico, productividad del agua.

\section{SUMMARY}

Water availability is the main environmental limitation for dry chili (Capsicum annuum L.) production in the North Central Area of México. The aim of this study was to compare four intensities of irrigation deficit (DI) against full irrigation (FI), in terms of fruit yield, dry chili quality, and irrigation water use efficiency (IWUE) in 'Mirasol' chili grown in a semiarid zone. The DI treatments were: $85 \%$ (DI 85), $70 \%$ (DI 70), $55 \%$ (DI 55), and $40 \%$ of FI (DI 40). Average water savings in DI 85, DI 70, DI 55 and DI 40 were $8,16,23$ and $30 \%$, respectively. The DI 40 treatment produced the highest percentage of marketable fruit, with similar yields to FI in two out of three years that the treatments were evaluated. The DI 40 showed increased IWUE, and reduced irrigation water volume by $1520 \mathrm{~m}^{3} \mathrm{ha}^{-1}$ compared to FI. Therefore, this treatment might be a water-saving alternative for this semiarid region and for other ones facing groundwater over-exploitation, without compromising dry chili quality. However, further studies regarding DI for this crop are needed in areas with low or no rainfall.

Index words: Capsicum annuum, water deficit, water productivity.

\section{INTRODUCCIÓN}

En las zonas áridas y semiáridas del norte de México, incluida la zona productora de chile 'Mirasol' (Capsicum annuum L.) para deshidratado, el agua es un recurso escaso. Esta condición se acentúa debido a un déficit en la recarga de los acuíferos, como consecuencia de que el régimen pluvial ha disminuido, de la sobre explotación del agua del subsuelo y de la baja eficiencia tecnológica en la aplicación del agua de riego en las unidades de producción (Galindo y Cabañas, 2006; Echavarría et al., 2009). Por tanto, es necesaria la optimización en el uso y manejo del agua de riego para lograr la sostenibilidad de los cultivos básicos y hortícolas (CNA, 2008).

El riego reducido es una estrategia que se ha propuesto para el ahorro de agua y para incrementar la eficiencia de ésta en el riego, sin afectar el rendimiento y calidad de los productos frescos (Kang et al., 2001; Dorji et al., 2005; Serna-Pérez et al., 2008; 2011). El riego deficitario (RD) es una estrategia de irrigación en la que se humedece todo el sistema radical con un volumen de agua menor a la evapotranspiración prevaleciente (Goldhamer et al., 2006). Esta estrategia ha sido aplicada a cultivos perennes (Behboudian y Mills, 1997) y anuales (Dorji et al., 2005; Liu et al., 2006). Sin embargo, el RD produce efectos variados en el rendimiento de los cultivos (Kang et al., 1998; Tang et al., 2005). Por ejemplo, el RD redujo en $34 \%$ el rendimiento de fruto fresco de chile Ancho con respecto al rendimiento con riego completo (RC); no obstante, ambos tratamientos produjeron un rendimiento de fruto seco similar (Dorji et al., 2005). En términos de calidad, el RD ha incrementado la concentración de sólidos solubles totales y mejorado el color de epidermis del fruto (Dorji et al., 2005; GonzálezDugo et al., 2007).

Con excepción de los estudios de chile 'Mirasol' bajo riego parcial de la raíz (Serna-Pérez et al., 2008; 2011), el uso del RD en la producción de chile 'Mirasol' ha recibido poca atención en las zonas productoras del país. En consecuencia, el objetivo de este estudio fue comparar cuatro intensidades de riego deficitario (RD) con el riego completo (RC), con base en el rendimiento, la calidad de fruto seco, y la eficiencia del uso del agua de riego (EUAR) en chile 'Mirasol' cultivado en una zona semiárida del centro norte de México. La hipótesis fue que al menos uno de los tratamientos de RD mantiene el rendimiento y calidad de chile deshidratado en comparación con RC, y mejora la eficiencia del uso del agua de riego en chile 'Mirasol'.

\section{MATERIALES Y MÉTODOS}

El experimento se hizo durante las estaciones de crecimiento de 2008 a 2010 en el Campo Experimental 
Zacatecas (CEZAC), Calera, Zacatecas, México (220 54' LN, $102^{\circ} 39^{\prime} \mathrm{LO}$ ), a una altitud de $2197 \mathrm{~m}$, con temperatura media anual de $14.6^{\circ} \mathrm{C}$, precipitación pluvial media anual de $416 \mathrm{~mm}$, y evaporación promedio anual de $1609 \mathrm{~mm}$. Las mayores diferencias entre lluvia y evaporación se registran de diciembre a la segunda decena de junio (Medina y Ruiz, 2004). El suelo es un Kastanozem de textura franco-arenosa con $\mathrm{pH}$ de 7.5 y un contenido de materia orgánica de $0.8 \%$.

Las plántulas de chile 'Mirasol' se desarrollaron en invernadero y se trasplantaron $75 \mathrm{~d}$ después de la siembra, cuando tenían entre 12 y 14 hojas, el 26, 11 y 24 de abril de 2008, 2009 y 2010, respectivamente. Las plantas se establecieron en surcos de $76 \mathrm{~cm}$, a hilera sencilla y a una densidad de 44 mil plantas ha-1 ${ }^{-1}$ El manejo del cultivo y control de plagas y enfermedades se efectuaron conforme al protocolo del CEZAC (Bravo et al., 2006). El cultivo se fertirrigó con $200 \mathrm{~N}-75 \mathrm{P}-100 \mathrm{~K}-60 \mathrm{Ca}$, de la siguiente manera: a los $20 \mathrm{~d}$ después del trasplante (DDT) se aplicó una fertilización de fondo con $40 \mathrm{~N}-30 \mathrm{P}-40 \mathrm{~K}$, seguida de dos fertilizaciones con $60 \mathrm{~N}-22.5 \mathrm{P}-30 \mathrm{~K}$ a los 50 y 70 DDT; respectivamente. Finalmente, durante el cuajado y desarrollo del fruto se aplicaron dos fertilizaciones con 20N-00P-00K-30Ca a los 80 y 100 DDT, respectivamente.

Se evaluaron los tratamientos de RC y cuatro intensidades de $\mathrm{RD}$, los cuales consistieron en la aplicación de un porcentaje del agua aplicada en el tratamiento de RC (Dorji et al., 2005). Los tratamientos de RD fueron: $85 \%\left(\mathrm{RD}_{85}\right)$; $70 \%\left(\mathrm{RD}_{70}\right) ; 55 \%\left(\mathrm{RD}_{55}\right) ;$ y $40 \% \operatorname{del} \mathrm{RC}\left(\mathrm{RD}_{40}\right)$. Los experimentos se condujeron en un diseño experimental en bloques completos aleatorizados con cuatro repeticiones. La unidad experimental fue de 8 surcos de 10 m de longitud, de los cuales sólo los cuatro surcos centrales fueron utilizados para registrar las variables de respuesta.

Al trasplante se aplicó una lámina de riego para recuperar la humedad del suelo hasta capacidad de campo $\left(\theta_{\mathrm{CC}}=0.28 \mathrm{~cm}^{3} \mathrm{~cm}^{-3} \mathrm{y} \theta_{\text {Inicial }} \sim 0.1 \mathrm{~cm}^{3} \mathrm{~cm}^{-3}\right)$. Se aplicaron de cuatro a seis riegos de magnitud igual a RC para establecer el cultivo, y posteriormente se dio inicio a la aplicación de los tratamientos con RD a los 61, 35 y 40 DDT, para 2008, 2009 y 2010, respectivamente. El agua de riego se aplicó por cintilla dos veces por semana en cada tratamiento, con 3 y $4 \mathrm{~d}$ de diferencia entre riegos. La lámina de riego para RC se estimó mediante un balance hídrico a partir de la diferencia entre la evapotranspiración del cultivo $(E T c)$ y la precipitación efectiva ( $P P e f)$ acumulada en cada periodo de riego. La ETc se estimó a partir de la evaporación libre del tanque evaporímetro tipo A $(E v)$, coeficiente del tanque $(K p=0.75)$ y coeficientes de cultivo $(K c)$, los cuales varían con el mes del año y con la etapa fenológica del cultivo (Bravo y Mojarro, 2006). La relación utilizada fue
$E T c=0.75 \times E v \times K c$. En la estimación de PPef se consideró aquellos eventos lluviosos individuales mayores a 5 $\mathrm{mm}$ que se acumularon entre riegos, y el total se multiplicó por el factor 0.75 (Serna-Pérez et al., 2011). La información climatológica se registró automáticamente en la estación meteorológica del CEZAC.

La densidad de raíces (DDR; g de materia seca de raíz/kg de suelo seco) de la planta de chile se estimó a los 120 DDT mediante la técnica descrita por Serna-Pérez et al. (2011), sólo en 2010. Al final del experimento se determinó el rendimiento y calidad de chile seco por unidad experimental. La calidad del fruto seco se evaluó de acuerdo con cuatro categorías comerciales según la norma Mexicana para chiles secos enteros (Zegbe-Domínguez et al., 2012): primera $(\mathrm{P})$, segunda $(\mathrm{S})$, tercera $(\mathrm{T})$ y manchados $(\mathrm{M})$. Se registró el peso por categoría y se determinó la distribución relativa por categoría (\%) con base en el rendimiento total por unidad experimental. La eficiencia en el uso del agua de riego (EUAR, $\mathrm{kg} \mathrm{ha}^{-1} \mathrm{~mm}^{-1}$ ) se estimó con la relación entre el rendimiento por unidad de área y la lámina de riego aplicada por tratamiento.

La información se analizó con un modelo lineal en bloques completos al azar con el procedimiento GLM del sistema de análisis estadístico SAS (SAS Institute, 2002). Antes del análisis, el porcentaje de cada categoría de calidad de fruto fue trasformado a valor de arco-seno. Los valores medios por tratamiento de las variables transformadas, se indican después de su re-transformación. La diferencia mínima significativa de Fisher $(\mathrm{P} \leq 0.05)$ se utilizó como criterio en la comparación de medias de tratamiento. La distribución porcentual de las categorías de calidad de fruta fueron similares entre años; por tanto cada categoría se analizó como una serie de experimentos. El rendimiento y la EUAR se analizaron por año de evaluación.

\section{RESULTADOS Y DISCUSIÓN}

Los tratamientos de RD fueron teóricamente diseñados para aplicar $85 \%$, $70 \%$, $55 \%$ y $40 \%$ del RC. Sin embargo, el volumen de agua empleada en promedio fue en realidad de $92 \%, 84 \%, 77$ \% y $70 \%$ de RC (Cuadro 1). Esto se debió a que durante el establecimiento del cultivo se usaron láminas de riego equivalentes a RC antes de aplicar los tratamientos, las que totalizaron 261, 123 y $141 \mathrm{~mm}$ en los ciclos 2008, 2009 y 2010 , respectivamente.

Durante el periodo de estudio la proporción de frutos de primera, segunda y tercera fue modificada por los tratamientos de riego (Cuadro 2). A pesar del déficit hídrico al que el cultivo fue sometido, el tratamiento $\mathrm{RD}_{40}$ indujo mayor proporción de frutos de primera y redujo la proporción de frutos secos de segunda y tercera en comparación 
con el resto de tratamientos. En cambio, $\mathrm{RD}_{85} \mathrm{y} \mathrm{RD}_{70}$ tendieron a reducir la proporción de frutos manchados.

El rendimiento total de chile seco fue diferente $(\mathrm{P}<0.05)$ entre tratamientos en los tres ciclos de cultivo (Cuadro 3).

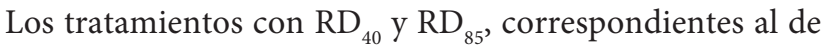
mayor y de menor déficit hídrico, respectivamente, produjeron rendimientos similares al RC en 2008. En 2009, los rendimientos en los tratamientos con $\mathrm{RD}$ fueron menores al rendimiento obtenido con RC. En 2010, el rendimiento en los tratamientos $\mathrm{RD}_{70} \mathrm{y} \mathrm{RD}_{40}$ fue similar al observado en RC. El rendimiento promedio producido en la evaluación de 2009 fue menor al registrado en 2008 y 2010.

La eficiencia en el uso del agua de riego (EUAR) fue consistentemente mayor $(\mathrm{P}<0.05)$ en $\mathrm{RD}_{40}($ Cuadro 3$)$ que en el resto de tratamientos de riego, en los ciclos 2008 y 2010. Los promedios más bajos en EUAR ocurrieron en el año 2009 debido a la reducción en el rendimiento ocurrida en ese año, y a la aplicación de un mayor volumen de riego durante la estación de crecimiento del cultivo (Cuadro 1). Con excepción del 2010, la EUAR más baja se observó en $\mathrm{RD}_{55}$, debido a que produjo los rendimientos más bajos.

Se ha reportado que el déficit hídrico reduce el rendimiento de fruto (Katerji et al., 1993; Dorji et al., 2005) y puede llegar a afectar su calidad comercial (Khah et al., 2007). Sin embargo, en este estudio los tratamientos $\mathrm{RD}_{85}$, $\mathrm{RD}_{70} \mathrm{y} \mathrm{RD}_{40}$ no redujeron la proporción de frutos de primera en comparación con el RC. Los tratamientos con RD en general redujeron el rendimiento proporcionalmente a la reducción en el volumen de agua de riego aplicada. No

Cuadro 1. Lámina de riego aplicada durante la estación de crecimiento en el riego completo (RC) y en los tratamientos con riego deficitario (RD) en chile 'Mirasol' para secado.

\begin{tabular}{lccc}
\hline \multirow{2}{*}{ Tratamientos de riego } & \multicolumn{3}{c}{ Lámina de agua $(\mathrm{mm})$} \\
\cline { 2 - 4 } & 2008 & 2009 & 2010 \\
$\mathrm{RC}$ & 409 & 567 & 527 \\
$\mathrm{RD}_{85}$ & 391 & 510 & 477 \\
$\mathrm{RD}_{70}$ & 373 & 452 & 427 \\
$\mathrm{RD}_{55}$ & 355 & 414 & 376 \\
$\mathrm{RD}_{40}$ & 338 & 382 & 327 \\
\hline
\end{tabular}

$\mathrm{RC}=$ riego completo; $\mathrm{RD}_{85}, \mathrm{RD}_{70}, \mathrm{RD}_{55} \mathrm{y} \mathrm{RD}_{40}=$ riego deficitario con $85 \%, 70 \%, 55 \%$ y $40 \%$ del RC, respectivamente. La precipitación efectiva (mm) fue de 267, 143 y 205 para 2008, 2009 y 2010, respectivamente.

Cuadro 2. Distribución porcentual promedio del peso de frutos secos de chile 'Mirasol' en función de tratamientos con riego deficitario (RD) y riego completo $(\mathrm{RC})$ durante el estudio de 2008 a 2010.

\begin{tabular}{|c|c|c|c|c|}
\hline \multirow{2}{*}{ Tratamientos de riego } & \multicolumn{4}{|c|}{ Distribución del peso de fruto seco (\%) } \\
\hline & $\mathrm{P}$ & $\mathrm{S}$ & $\mathrm{T}$ & M \\
\hline $\mathrm{RC}$ & $61.2 \mathrm{bc}$ & $14.8 \mathrm{ab}$ & $13.7 \mathrm{ab}$ & $10.3 \mathrm{a}$ \\
\hline $\mathrm{RD}_{85}$ & $62.8 \mathrm{abc}$ & $15.9 \mathrm{ab}$ & $14.2 \mathrm{ab}$ & $7.1 \mathrm{a}$ \\
\hline $\mathrm{RD}_{70}$ & $65.1 \mathrm{ab}$ & $15.1 \mathrm{ab}$ & $12.3 \mathrm{ab}$ & $7.5 \mathrm{a}$ \\
\hline $\mathrm{RD}_{55}$ & $57.6 \mathrm{c}$ & $16.6 \mathrm{a}$ & $15.0 \mathrm{a}$ & $10.8 \mathrm{a}$ \\
\hline $\mathrm{RD}_{40}$ & $67.4 \mathrm{a}$ & $13.1 \mathrm{~b}$ & $11.1 \mathrm{~b}$ & $8.4 \mathrm{a}$ \\
\hline
\end{tabular}

Letras diferentes en una columna indican diferencias significativas ( $\mathrm{P} \leq 0.05)$. Los valores medios en porcentaje fueron transformados a arco seno antes del análisis. $\mathrm{RC}=$ riego completo; $\mathrm{RD}_{85}, \mathrm{RD}_{70}, \mathrm{RD}_{55}$ y $\mathrm{RD}_{40}=$ riego deficitario con $85 \%, 70 \%, 55 \%$ y $40 \%$ del $\mathrm{RC}$, respectivamente. $\mathrm{P}, \mathrm{S}, \mathrm{T}$ y $\mathrm{M}=$ frutos de primera, segunda, tercera y manchados, respectivamente.

Cuadro 3. Rendimiento total de frutos secos de chile 'Mirasol' y eficiencia en el uso del agua de riego (EUAR) en función de riego completo (RC) y tratamientos con riego deficitario (RD).

\begin{tabular}{|c|c|c|c|c|c|c|}
\hline \multirow{2}{*}{ Tratamientos de riego } & \multicolumn{3}{|c|}{ Rendimiento ( $\mathrm{t} \mathrm{ha}^{-1}$ ) } & \multicolumn{3}{|c|}{$\operatorname{EUAR}\left(\mathrm{kg} \mathrm{ha}^{-1} \mathrm{~mm}^{-1}\right)$} \\
\hline & 2008 & 2009 & 2010 & 2008 & 2009 & 2010 \\
\hline $\mathrm{RC}$ & $3.3 \mathrm{a}$ & $2.5 \mathrm{a}$ & $2.6 \mathrm{a}$ & $8.0 \mathrm{~b}$ & $4.4 \mathrm{ab}$ & $4.9 \mathrm{c}$ \\
\hline $\mathrm{RD}_{85}$ & $2.9 \mathrm{ab}$ & $2.1 \mathrm{~b}$ & $2.1 \mathrm{~b}$ & $7.3 \mathrm{bc}$ & $4.1 \mathrm{abc}$ & $4.4 \mathrm{c}$ \\
\hline $\mathrm{RD}_{70}$ & $2.7 \mathrm{bc}$ & $1.8 \mathrm{bc}$ & $2.7 \mathrm{a}$ & $7.2 \mathrm{bc}$ & $4.0 \mathrm{bc}$ & $6.3 \mathrm{~b}$ \\
\hline $\mathrm{RD}_{55}$ & $2.3 c$ & $1.5 \mathrm{c}$ & $2.0 \mathrm{~b}$ & $6.4 \mathrm{c}$ & $3.7 c$ & $5.4 \mathrm{bc}$ \\
\hline $\mathrm{RD}_{40}$ & $3.2 \mathrm{ab}$ & $1.8 \mathrm{bc}$ & $2.5 \mathrm{ab}$ & $9.6 \mathrm{a}$ & $4.8 \mathrm{a}$ & $7.6 \mathrm{a}$ \\
\hline
\end{tabular}


obstante, el $\mathrm{RD}_{40}$ produjo rendimientos similares al RC en 2008 y 2010, y además en esos años alcanzó los mayores valores de EUAR.

Una característica que permite a las plantas sobrellevar eventos deficitarios de humedad es la capacidad del sistema radical para explorar mayores volúmenes de suelo. Kulkarni y Phalke (2009) encontraron que la densidad lateral de raíces, entre otras variables estudiadas, mostró una buena correlación positiva con el rendimiento en C. annuum $\mathrm{L}$. En este estudio, la densidad de raíces (DDR) en las plantas bajo $\mathrm{RD}_{40}$ y $\mathrm{RD}_{55}$ fue mayor que en los otros tratamientos, lo cual en parte podría explicar la similitud de rendimientos entre $\mathrm{RD}_{40}$ y RC. Los valores de DDR (g de MS de raíz/ $\mathrm{kg}$ de suelo seco) para $\mathrm{RC}, \mathrm{RD}_{85}$ y $\mathrm{RD}_{70}$ fueron $1.21,1.07 \mathrm{y}$ 1.19 , menores $(\mathrm{P}<0.05)$ que los de $\mathrm{RD}_{55}$ y $\mathrm{RD}_{40}$ con $1.87 \mathrm{y}$ 2.32 .

Los volúmenes promedio de agua aplicados en los tres años en $\mathrm{RC}$ y $\mathrm{RD}_{40}$ fueron de $5010 \pm 821 \mathrm{~m}^{3} \mathrm{ha}^{-1}$ y 3490 $\pm 291 \mathrm{~m}^{3} \mathrm{ha}^{-1}$, respectivamente. El primer volumen de riego es similar al reportado por Khah et al. (2007), quienes determinaron un volumen total de $5560 \mathrm{~m}^{3} \mathrm{ha}^{-1}$ como el adecuado para obtener el mayor rendimiento comercial en riego por goteo. En este estudio, mediante la aplicación del $\mathrm{RD}_{40}$ fue posible reducir en promedio el volumen de agua de riego aplicada en $1520 \mathrm{~m}^{3} \mathrm{ha}^{-1}$ con respecto al RC. No obstante que este tratamiento podría ser una alternativa para el ahorro de agua en la región Norte Centro de México, más investigación sobre el efecto de $\mathrm{RD}$ es necesaria para explicar el comportamiento en la producción de chile para secado cuando la precipitación efectiva tiende a reducirse o a ser nula.

\section{CONCLUSIONES}

De los tratamientos con riego deficitario, el de $40 \%$ $\left(\mathrm{RD}_{40}\right)$ produjo chiles de mejor calidad comercial y con rendimiento similar que el riego de $100 \%$ (RC), en dos de los tres años de evaluación. Además, durante el período de estudio $\mathrm{RD}_{40}$ mantuvo en promedio la más alta eficiencia en el uso del agua de riego. Lo anterior derivó en un ahorro de $1520 \mathrm{~m}^{3} \mathrm{ha}^{-1}$ en relación con el RC. Sin embargo, el riego deficitario requiere de más investigación para elucidar el comportamiento del cultivo cuando la precipitación efectiva se reduce o es nula durante la estación de desarrollo.

\section{AGRADECIMIENTOS}

A los Sres. Miguel Juárez Aguilar y Pedro Castañón Hernández por la asistencia técnica. AL Consejo Nacional de Ciencia y Tecnología (No. ref.: SNI-53131) y a la Fundación Produce Zacatecas, A.C. (No. ref.: 097/FPZ/2005), por el financiamiento parcial.

\section{BIBLIOGRAFÍA}

Behboudian M H, T M Mills (1997) Deficit irrigation in deciduous orchards. Hort. Rev. 21:105-131

Bravo A G, G Galindo, M D Amador (2006) Tecnología de Producción de Chile Seco. SAGARPA-INIFAP-CIRNOC, Campo Experimental Zacatecas. Libro Técnico No. 5. 222 p.

Bravo A G, F Mojarro (2006) Riego por goteo y fertirrigación. In: Tecnología de Producción de Chile Seco. G Bravo A, G Galindo, M D Amador (eds). SAGARPA-INIFAP-CIRNOC-Campo Experimental Zacatecas. Libro Técnico No. 5. pp:61-76.

Comisión Nacional del Agua (2008) Estadísticas del Agua en México 2008. Secretaría del Medio Ambiente y Recursos Naturales (ed). México, D.F. 233 p.

Dorji K, M H Behboudian, J A Zegbe-Domínguez (2005) Water relations, growth, yield, and fruit quality of hot pepper under deficit irrigation and partial rootzone drying. Sci. Hort. 104:137149.

Echavarría F G, G Medina, A F Rumayor, A Serna, H Salinas, J G Bustamante (2009) Diagnóstico de los Recursos Naturales para la Planeación de la Intervención Tecnológica y el Ordenamiento Ecológico. SAGARPA-INIFAP-CIRNOC-Campo Experimental Zacatecas. Libro Técnico No. 10. 174 p.

Galindo G, B Cabañas (2006) El cultivo de chile en Zacatecas. In: Tecnología de Producción de Chile Seco. G Bravo A, G Galindo, M D Amador (eds). SAGARPA-INIFAP-CIRNOC-Campo Experimental Zacatecas. Libro Técnico No. 5. pp:5-18.

Goldhamer D A, M Viveros, M Salinas (2006) Regulated deficit irrigation in almonds: effects of variation in applied water and stress timing on yield and yield components. Irrig. Sci. 24:101-114.

González-Dugo V, F Orgaz, E Fereres (2007) Responses of pepper to deficit irrigation for paprika production. Sci. Hort. 114:77-82.

Katerji N, M Mastrorilli, A Hamdy (1993) Effects of water stress at different growth stages on pepper yield. Acta Hort. 335:165-171.

Kang S, Z Liang, W Hu, Y J Zhang (1998) Water use efficiency of controlled alternate irrigation on root-divided maize plants. Agric. Water Manage. 38:69-76.

Kang S, L Zhang, X Hu, Z Li, P Jerie (2001) An improved water use efficiency for hot pepper grown under controlled alternate drip irrigation on partial roots. Sci. Hort. 89:257-267.

Khah E M, K Koukoufikis, A G Mavromatis, D Chachalis, C K Goulas (2007) The effect of different techniques on plant performance and seed quality in relation to fruit maturation and storage of different genotypes of pepper (Capsicum annuum var. annuиm L.). J. Food Agric. Environ. 5:159-163.

Kulkarni M, S Phalke (2009) Evaluating variability of root size system and its constitutive traits in hot pepper (Capsicum annuum L.) under water stress. Sci. Hort. 120:159-166.

Liu F, A Shahnazari, M N Andersen, S E Jacobsen, C Jensen (2006) Effects of deficit irrigation (DI) and partial root drying (PRD) on gas exchange, biomass partitioning, and water use efficiency in potato. Sci. Hort. 109:113-117.

Medina G, A Ruíz (2004) Estadísticas Climatológicas Básicas del Estado de Zacatecas (Período 1961 - 2003). SAGARPA-INIFAPCIRNOC-Campo Experimental Zacatecas. Libro Técnico No. 3. $40 \mathrm{p}$.

SAS (2002) Statistical Analysis System. SAS software version 9.1. SAS Institute, Inc. Cary, NC, USA.

Serna-Perez A, J A Zegbe, J Mena-Covarrubias, S Rubio-Díaz (2008) Sistemas de manejo para la producción sustentable de chile seco cv. 'Mirasol'. Rev. Fitotec. Mex. 31 (Núm. Especial 3): 1-44.

Serna-Pérez A, J A Zegbe, J Mena-Covarrubias (2011) Rendimiento y calidad de chile seco 'Mirasol' cultivado bajo riego parcial de la raíz. Rev. Chapingo S. Hort. 17 (Especial 1):19-24.

Tang L S, Y Li, J Zhang (2005) Physiological and yield responses of cotton under partial rootzone drying. Field Crops Res. 94:214-223.

Zegbe-Domínguez J A, J Mena-Covarrubias, R D Valdez-Cepeda, M D Amador-Ramírez, G Esparza-Frausto (2012) Importancia, diversidad genética y situación actual del cultivo del chile en México: In: El Cultivo del Chile en México. Tendencias de Producción y Problemas Fitosanitarios Actuales. J A ZegbeDomínguez, R D Valdez-Cepeda, A Lara-Herrera (eds). Universidad Autónoma de Zacatecas. Zacatecas, México. pp:9-47. 